\section{Comparison of two different lung ultrasound imaging protocols in COVID-19 pneumonia}

\author{
Korgün Ökmen, Durdu Kahraman Yildiz, Emel Soyaslan, IIlkay Ceylan, Halil Erkan Sayan, \\ Cihan Sedat Aytünür
}

Department of Anesthesiology and Reanimation, University of Health Sciences, Bursa Yuksek Ihtisas Training and Research Hospital, Bursa, Turkey

Purpose: The aim of this study was to determine the effectiveness of two different lung ultrasonography (LUS) methods that can be used in the diagnosis of coronavirus disease 2019 (COVID-19) and to investigate their correlations with computed tomography (CT).

Methods: In this prospective, randomized, and single-blind study, 60 patients with COVID-19 were included. The patients were randomized to either the 12-zone LUS group $(n=30)$ or the 14zone LUS group $(n=30)$. The correlation between LUS and thoracic CT scores was evaluated. As a secondary outcome measure, the characteristic features of the findings of thoracic CT and LUS were examined.

Results: The study was completed with a total of 59 patients. Moderate and high correlations were found between the total CT and LUS scores in the 12-zone and 14-zone study groups. There were no statistically significant differences in the lesion types detected in patients using LUS and CT ( $P>0.05)$. The left lung lower lobe CT scores were statistically significantly lower in the 14-zone study group than in the 12-zone group $(P=0.019)$. The left lower lobe $C T$ and LUS scores were highly correlated in the 14-zone group $(P<0.001, r=0.902)$.

Conclusion: The results of our study indicated that the two different LUS examination methods performed in different patients had similar findings in terms of the diagnosis and their correlations with $\mathrm{CT}$ results.

Keywords: COVID-19; Lung ultrasound; Computed tomography; Pneumonia

Key points: There is a strong role of lung ultrasonography (LUS) in the diagnosis of pneumonia associated with coronavirus disease 2019. The results of different LUS examination methods had similar findings in terms of diagnosis and correlation with computed tomography results.

\section{Introduction}

Severe acute respiratory syndrome coronavirus 2 infection, which causes coronavirus disease 2019 (COVID-19), has continued as a pandemic posing major health issues in many countries worldwide for over a year [1]. COVID-19 can be transmitted by droplets. It can be treated on an outpatient

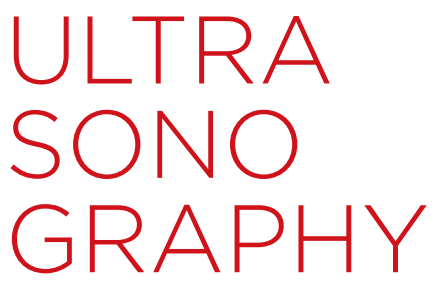

ORIGINAL ARTICLE

https://doi.org/10.14366/usg.21095 pISSN: 2288-5919 • elSSN: 2288-5943 Ultrasonography 2022;41:212-221

Received: April 30, 2021

Revised: July 13, 2021

Accepted: July 13, 2021

Correspondence to: Korgün Ökmen, MD, PhD, Department of Anesthesiology and Reanimation, University of Health Sciences, Bursa Yuksek Ihtisas Training and Research Hospital, Mimar Sinan Mah. Emniyet Street Yıldırım, Bursa 16130, Turkey

Tel. +90-224-2955000

Fax. +90-224-295-5497

E-mail: korgunokmen@gmail.com

This is an Open Access article distributed under the terms of the Creative Commons Attribution NonCommercial License (http://creativecommons.org/ licenses/by-nc/4.0/) which permits unrestricted noncommercial use, distribution, and reproduction in any medium, provided the original work is properly cited.

Copyright (C) 2022 Korean Society of Ultrasound in Medicine (KSUM)

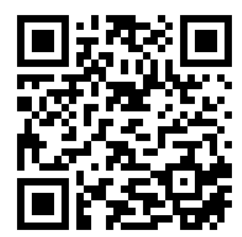

How to cite this article:

Ökmen K, Yildiz DK, Soyaslan E, Ceylan I, Sayan $\mathrm{HE}$, Aytünür CS. Comparison of two different lung ultrasound imaging protocols in COVID-19 pneumonia. Ultrasonography. 2022 Jan;41(1):212-221. 
basis in many patients, although approximately $20 \%$ may need to be hospitalized. COVID-19 primarily involves respiratory system symptoms, which progress from mild symptoms to pneumonia and acute respiratory distress syndrome, with the risk of severe outcomes depending on patients' demographic characteristics and comorbidities [2]. Lung imaging methods are important in the context of COVID-19, as the disease mainly affects the respiratory tract, and pulmonary findings are common. In addition to the conventional methods of computed tomography (CT) and chest radiography, lung ultrasonography (LUS) can be used in the diagnosis and follow-up of COVID-19 [3]. The sensitivity of CT was found to be $90 \%-97.2 \%$. While the early-stage finding specific to COVID-19 is ground-glass opacity, this finding may be accompanied by interlobular septal thickening and a paving stone appearance in advanced stages [4-6].

The role of LUS during the COVID-19 pandemic has been described in various studies in the literature, which have demonstrated that it can be used for diagnosis and follow-up [7]. The main advantages of LUS over CT have been reported in the literature as its portability, the lack of a radiation dose, the ability to perform LUS without the need for auxiliary personnel, and its reproducibility [7]. The ultrasonographic findings related to COVID-19 are similar to those of viral pneumonia; however, pleural irregularities, abnormalities in B lines, and unique findings have also been detected [8]. As a result of differences in the areas observed in previous studies and the type of probe used, standardization has yet to be achieved. A few authors have presented methods and discussed the necessity of administering standard procedures [9]. Evaluation of 12- and 14-zone study areas are two prominent methods. It is thought that the 14-zone examination method can provide a more detailed examination of the posterior lung regions. The fact that pneumonia caused by COVID-19 mostly affects the lower lung regions may underscore the importance of this method [9].

The present study aimed to determine the effectiveness of two different LUS methods for the diagnosis of COVID-19 and to investigate their correlations with $\mathrm{CT}$.

\section{Materials and Methods}

\section{Compliance with Ethical Standards}

This prospective, randomized, single-blind study included 65 patients who were admitted to the intensive care unit with the diagnosis of COVID-19, with approval from the ethics committee (No. 2021/0121). Informed consent was obtained from all individual participants included in the study. All procedures performed in studies involving human participants were in accordance with the ethical standards of the institutional and/or national research committee and with the 1964 Helsinki declaration and its later amendments or comparable ethical standards.

\section{Patients}

The study included patients between 18 and 85 years of age who were hospitalized in the intensive care unit, administered a reverse-transcription polymerase chain reaction (RT-PCR) test, and underwent thoracic CT due to suspected COVID-19. A medical history of lung operation, thoracic wall abnormalities, and interstitial lung disease were used as the exclusion criteria. Subsequently, 60 patients who agreed to participate in the study and provided written consent were randomized using a table of random numbers to the 12-zone $(n=30)$ and 14-zone $(n=30)$ groups (Fig. 1).

\section{Radiological Evaluation: CT Procedure}

CT imaging was performed on patients using a standard method in the supine position with a 128-row detector $(120 \mathrm{kVp}$, with a reference $\mathrm{mAs}$ setting of 81 , a range of 1.0-1.5, and a collimated width of $0.625-1.0 \mathrm{~mm}$ ) (SOMATOM Definition AS+, Siemens Healthineers, Erlangen, Germany) [10]. The evaluation of thoracic CT was reported by the radiology department, unaware of the LUS findings, and it was scored by physicians in the Anesthesiology and Reanimation clinic (DKY, ES, and CSA). Thin-section CT was used to determine the $\mathrm{CT}$ scores [11]. Individual calculations were made for each lung lobe. During the calculation, the finding of no lesions was scored as $0 \%$ (0 points, ) the presence of lesions involving 1\%-5\% of the lobe was scored as 1 point, the presence of lesions involving $5 \%-25 \%$ of the lobe was scored as 2 points, the presence of

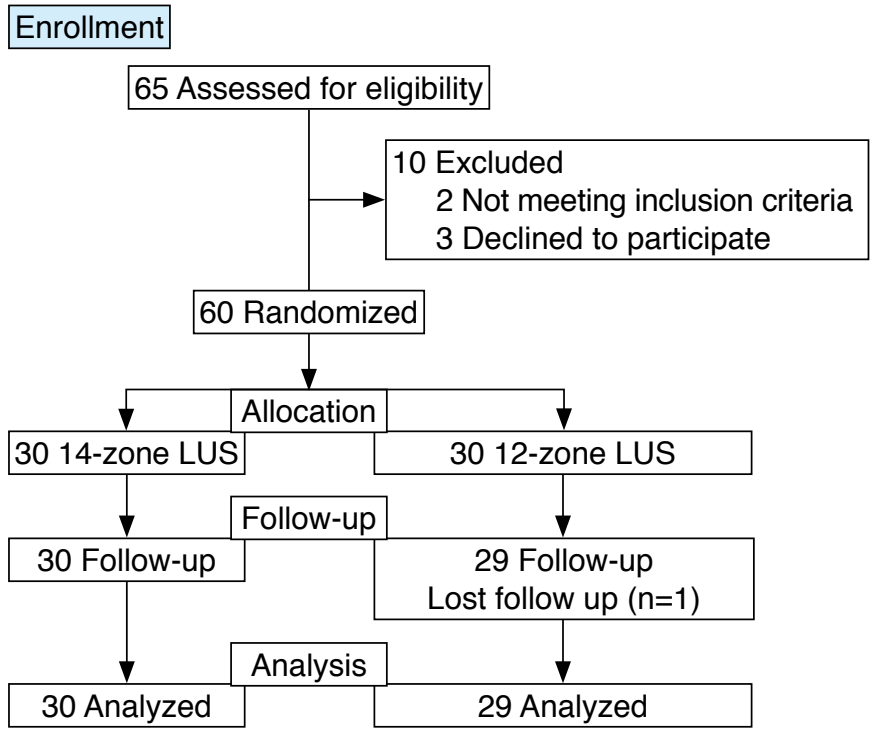

Fig. 1. CONSORT diagram. 
lesions involving $25 \%-50 \%$ of the lobe was scored as 3 points, the presence of lesions in more than half of the lobe $(50 \%-75 \%)$ was scored as 4 points, and the presence of lesions in more than $75 \%$ of the lobe was scored as 5 points. Calculations were made for each lung lobe, the right and left lungs, and both lungs [11].

\section{LUS Protocol}

LUS was performed with a convex ultrasound probe $(2-6 \mathrm{MHz})$ after protective measures were taken by the researcher, who was experienced in lung ultrasonography. The period of time between LUS and CT was 12 hours. The specialist who performed the LUS, did not have information about laboratory test values, the medical history of the patient, the results of the CT scan results, or the results of the RT-PCR test.

The examination of the 12 zones consisted of a 12-zone protocol including six zones in the right lung and six zones in the left lung, as previously defined [12] (Fig. 2, Supplementary Data 1). The measurements involved zones 1,3 , and 5 for the right upper lobe LUS score, zones 2 and 4 for the right middle lobe LUS score, zones 4 and 6 for the right lower lobe LUS score, zones 1, 3, and 5 for the left upper lobe LUS score, and zones 2, 4, and 6 for the left lower lobe LUS score.

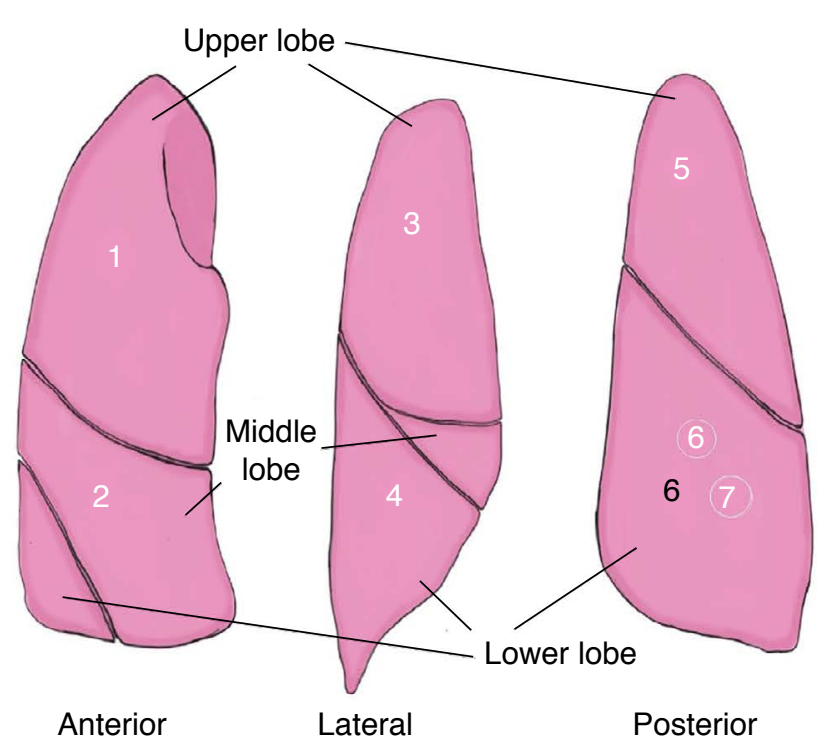

Right lung
The examination of the 14 zones consisted of a 14-zone protocol including seven zones in the right lung and seven zones in the left lung [9] (Fig. 2, Supplementary Data 1). The measurements involved zones 1, 3, and 5 for the right upper lobe LUS score, zones 2 and 4 for the right middle lobe LUS score, zones 4,6 , and 7 for the right lower lobe LUS score, zones 1,3 , and 5 for the left upper lobe LUS score, and zones 2, 4, 6, and 7 for the left lower lobe LUS score. Pleural lines, B lines, consolidations, pleural effusion, and all abnormal findings were recorded during the examination of each zone. A score between 0 and 3 was given for each zone. In scoring, $A$ lines and less than three $B$ lines were scored as 0 points, conjoined $B$ lines in less than $50 \%$ of the intercostal region were scored as 1 point, B lines (white lung) covering more than $50 \%$ of the intercostal region was scored as 2 points, and the detection of consolidation or pleural effusion was scored as 3 points (Figs. 3, 4) [13].

\section{Outcome Measures}

The primary outcome measure was the correlation between the results of LUS and thoracic CT. The secondary outcome measures were the characteristic features of the findings on thoracic $\mathrm{CT}$ and LUS, as well as correlations between physical examination findings

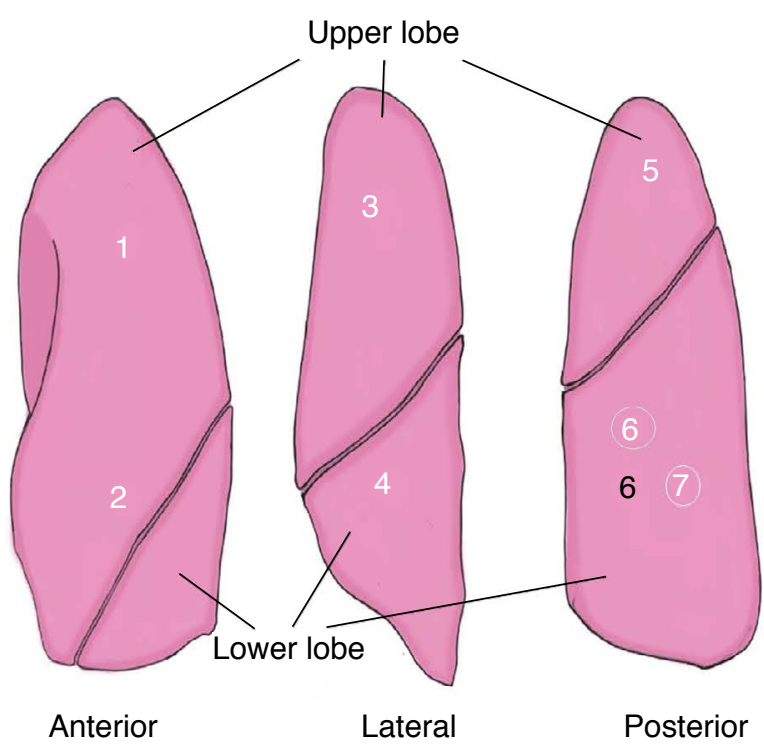

Fig. 2. Lung examination areas. Areas 1-5 are common areas for the 12- and 14-zone evaluations: number 1, upper part of the internipple line in the midclavicular line (area 1); number 2, lower part of the internipple line in the midclavicular line (area 2); number 3, upper part of the internipple line above the midaxillary line (area 3); number 4, lower part of the internipple line above the midaxillary line (area 4); number 5 , above the line joining the lower ends of the scapula in the paravertebral line (area 5). In the 12-zone group, the posterior-inferior lung region (black number 6) is below the line joining the lower ends of the scapula in the paravertebral line (area 6). In the 14-zone group, the posterior-inferior lung region is divided into multiple areas: the middle area along the paravertebral line at the inferior angle of the shoulder blade (white number 6, area 6) and the basal area along the paravertebral line above the curtain sign (white number 7, area 7). 
(Acute Physiology and Chronic Health Evaluation II score) and laboratory results (ferritin, C-reactive protein, saturated oxygen [\%], and lymphocyte count) with the LUS score.

\section{Statistical Analysis}

Data are presented as mean \pm standard deviation, and categorical data are presented as percentages. The distribution of the data was evaluated using the Shapiro-Wilk test. The independent-samples t-test was used for comparing the normally distributed data. The Pearson correlation test was used to evaluate the correlations between LUS scores and CT scores. The relationship was considered significant if the P-value was $<0.05$. SPSS version 22.0 (IBM Corp., Armonk, NY, USA) statistical software was used for data analysis.

\section{Power Analysis}

In studies that previously evaluated 12 zones of the lung, a strong correlation of 0.85 was found between the LUS score and the CT score [10]. Considering a bilateral value of $a=0.05$ and a power of $85 \%$, the number of patients required to detect a $10 \%$ difference between group correlations was determined to be 58 . It was planned to enroll 60 patients in the study, anticipating the possibility of some deaths during the study.

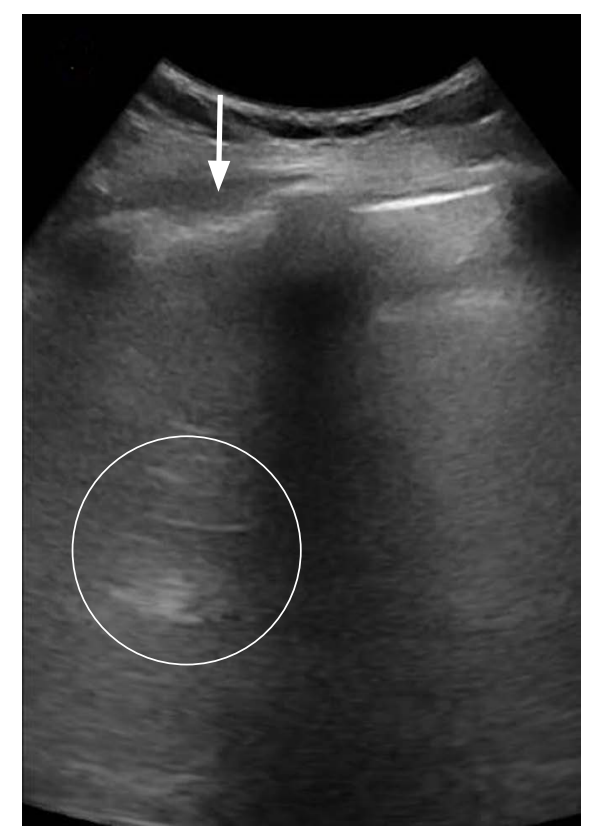

A
Fig. 3. Lung ultrasonography (LUS) and thoracic computed tomography (CT) images of a 54-year-old woman with coronavirus disease 2019 related pneumonia.

A-C. LUS image (A) of the irregular and thickened pleural line (arrow) of the suppleural lesion (arrow) detected on Thorax CT (B); at the level of the fourth-fifth intercostal space in the middle axillary region $(C)$ is shown.

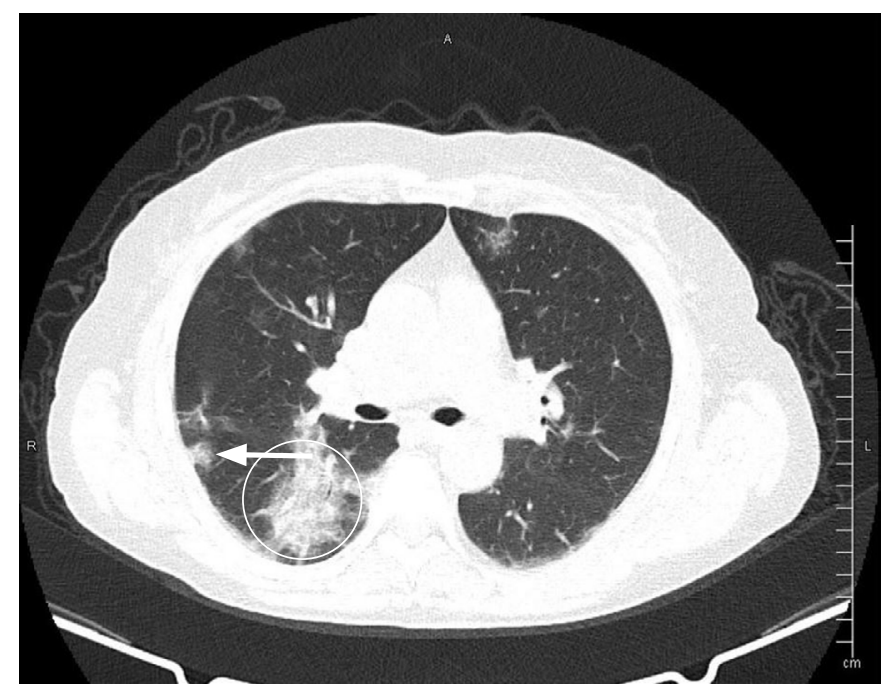

B

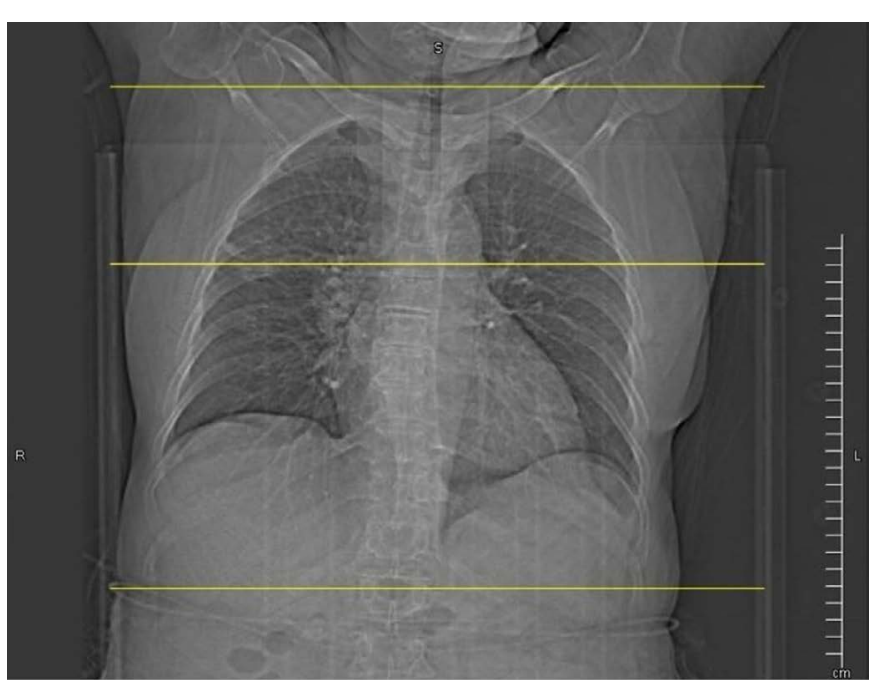

C 


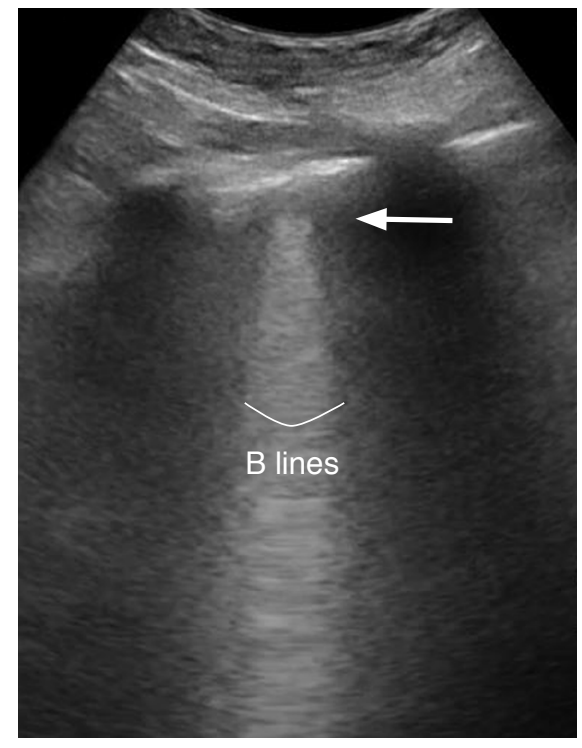

A

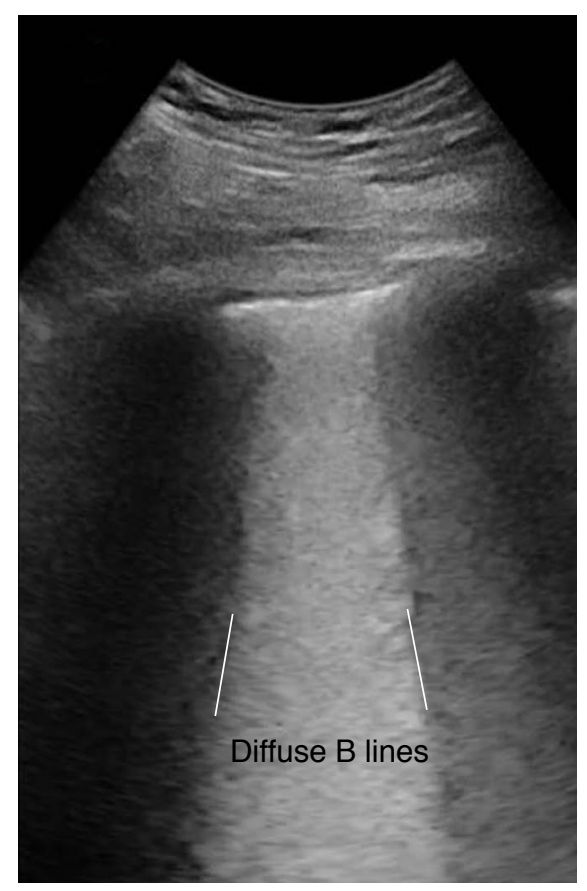

C

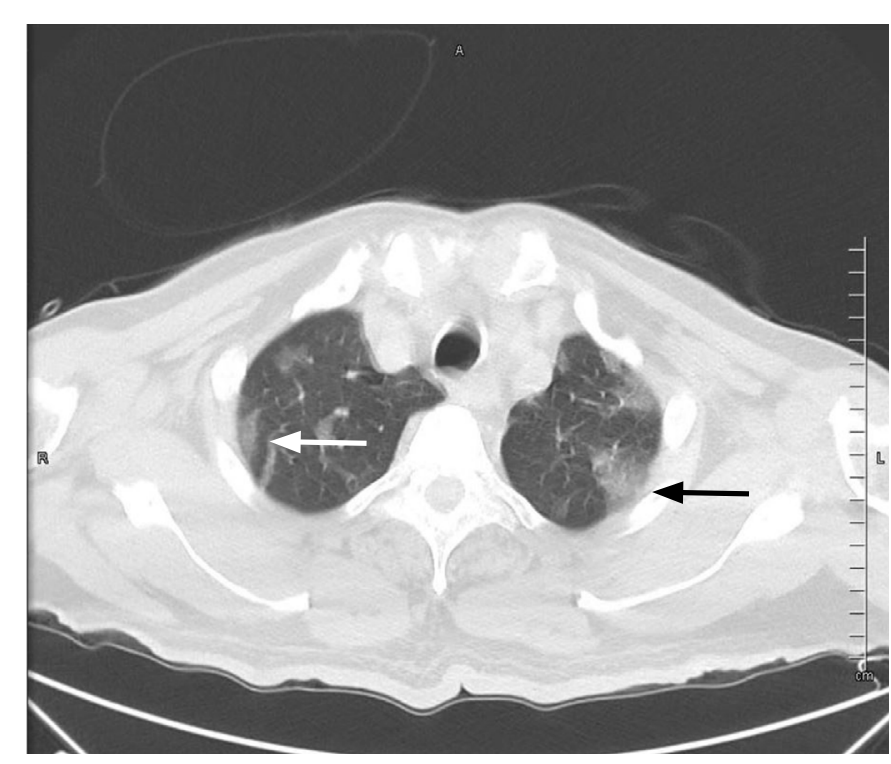

B

Fig. 4. Lung ultrasonography (LUS) and thoracic computed tomography (CT) images of a 64-year-old man with coronavirus disease 2019 related pneumonia.

A-C. An irregular and thickened pleural line starting from the subpleural hypoechoic area on LUS (A) is matched to a subpleural consolidation (white arrow) on CT (B). A ground-glass opacity (black arrow) detected in a larger area in the left hemithorax (B) is matched with diffuse $B$ lines of the white lung appearance covering the entire intercostal space on LUS (C).

\section{Results}

The study was completed with a total of 59 patients (14-zone examination group, $n=30 ; 12$-zone examination group, $n=29$ ) (Fig. 1), since one patient in the 12-zone examination group died before LUS was performed. The demographic characteristics, physical examination findings, and laboratory results of the patients are presented in Table 1. There was no statistically significant difference in the values between the groups $(P>0.05)$. All patients were classified as having been diagnosed with COVID-19 based on RT$P C R, C T$, and clinical findings before hospitalization.

When the thoracic $\mathrm{CT}$ results were examined, bilateral lung involvement was found in 54 patients and single lung involvement in five patients (three patients with right lung involvement, two patients with isolated left lung involvement). The CT and LUS findings of the 12-zone and 14-zone study groups are presented in Table 2. There were no statistically significant differences between the groups in terms of the lesion types detected in patients using 
Table 1. Comparison of the demographic characteristics of the groups

\begin{tabular}{|c|c|c|c|}
\hline Demographic characteristic & $\begin{array}{c}12 \text { Zones } \\
(\mathrm{n}=29)\end{array}$ & $\begin{array}{l}14 \text { Zones } \\
(n=30)\end{array}$ & P-value \\
\hline Age (year), mean $\pm S D$ & $62.1 \pm 17.9$ & $60.2 \pm 11.6$ & 0.816 \\
\hline $\begin{array}{l}\text { Body mass index }\left(\mathrm{kg} / \mathrm{m}^{2}\right) \text {, } \\
\text { mean } \pm \mathrm{SD}\end{array}$ & $26.4 \pm 6.8$ & $27.3 \pm 5.2$ & 0.320 \\
\hline Sex (male:female), n (\%) & $\begin{array}{l}19(65.5) / \\
10(34.5)\end{array}$ & $\begin{array}{l}20(66.7) / \\
10(33.3)\end{array}$ & 0.440 \\
\hline \multicolumn{4}{|l|}{$\begin{array}{l}\text { Physical exam and laboratory } \\
\text { results }\end{array}$} \\
\hline Apache score, mean \pm SD & $23.8 \pm 5.3$ & $22.4 \pm 5.6$ & 0.706 \\
\hline Ferritin $(\mathrm{ng} / \mathrm{mL})$, mean $\pm \mathrm{SD}$ & $1,126 \pm 326$ & $1,093 \pm 298$ & 0.704 \\
\hline C-reactive protein (mg/dL) & 90.3 & 86.4 & 0.354 \\
\hline $\mathrm{SpO}_{2}(\%)$, mean & 91.0 & 91.2 & 0.534 \\
\hline Lymphocytes $\left(\times 10^{9} / \mathrm{L}\right)$ & 0.58 & 0.61 & 0.969 \\
\hline \multicolumn{4}{|l|}{ RT-PCR, n (\%) } \\
\hline Positive & $20(68.9)$ & $21(70.0)$ & 0.805 \\
\hline Negative & $9(31.1)$ & $9(30.0)$ & 0.932 \\
\hline \multicolumn{4}{|l|}{ Comorbidities } \\
\hline No comorbidities & 9 & 11 & \\
\hline Hypertension & 13 & 12 & \\
\hline Diabetes & 14 & 10 & \\
\hline Cardiovascular disease & 5 & 3 & \\
\hline Renal disease & 1 & 1 & \\
\hline COPD & 1 & 1 & \\
\hline Other & 8 & 8 & \\
\hline
\end{tabular}

$\mathrm{SD}$, standard deviation; $\mathrm{SpO}_{2}$, saturated oxygen; RT-PCR, reverse-transcription polymerase chain reaction; COPD, chronic obstructive pulmonary disorder.
$P>0.05$. The total CT score of 59 patients included in the study was calculated as $11.9 \pm 6.3$. The total CT score of the left lung was found to be significantly lower in the 14-zone examination group (3.9 \pm 2.5$)$ than in the 12-zone group $(5.8 \pm 2.9, \mathrm{P}=0.013$ ) (Table 3). The CT score of the left lower lobe was found to be significantly lower in the 14-zone examination group than in the 12-zone group $P=0.019$ (Table 4). The correlations between the CT and LUS scores, both for the total scores and for each of the lung lobes, are presented in Tables 3 and 4 . Moderate and high correlations were found between the CT and LUS scores in the 12-zone and 14zone examination groups, respectively. The left lower lobe $\mathrm{CT}$ and LUS scores were highly correlated in the 14-zone group $(P<0.001$, $r=0.902$ ). The physical examination and laboratory results were moderately and strongly correlated, respectively, with the LUS score in both groups $(\mathrm{P}<0.05)$ (Table 5$)$.

$A$ receiver operating characteristic curve analysis showed that the area under the curve (AUC) for LUS was higher than that for RT-PCR for the detection of $\mathrm{CT}$ abnormalities in both groups. In the 12-zone group, the AUC of LUS was 0.895 (95\% confidence interval [CI], 0.711 to $1.000 ; P<0.001)$, while that of RT-PCR was $0.703(95 \%$ $\mathrm{Cl}, 0.457$ to $0.949 ; \mathrm{P}=0.132$ ), and in the 14-zone group, the AUC of LUS was $0.908(95 \% \mathrm{Cl}, 0.746$ to $1.000 ; P<0.001)$, while that of RT-PCR was 0655 (95\% Cl, 0.412 to 0.899; $P=0.132$ ) (Fig. 5).

For the total CT scores, inter-observer variability showed good consistency, with an intraclass correlation coefficient of $0.968(95 \%$ $\mathrm{Cl}, 0.933$ to 0.985$)$.

Table 2. CT and LUS findings

\begin{tabular}{|c|c|c|c|c|c|c|c|c|c|}
\hline \multicolumn{4}{|c|}{12 Zones } & \multicolumn{4}{|c|}{14 Zones } & \multirow{2}{*}{ P-value ${ }^{a)}$} & \multirow{2}{*}{ P-value ${ }^{b}$} \\
\hline CT finding & No. (\%) & LUS finding & №. (\%) & CT finding & No. (\%) & LUS finding & No. (\%) & & \\
\hline \multirow{3}{*}{$\begin{array}{l}\text { Ground-glass } \\
\text { opacities }\end{array}$} & $29(100)$ & Irregular pleural line & $27(93.1)$ & Ground-glass opacities & $30(100)$ & Irregular pleural line & $30(100)$ & 0.905 & 0.780 \\
\hline & & Compound B lines & $27(93.1)$ & & & Compound B lines & $26(86.7)$ & & 0.774 \\
\hline & & Diffuse B lines & $17(58.6)$ & & & Diffuse B lines & $14(46.7)$ & & 0.560 \\
\hline \multirow[t]{3}{*}{ Crazy paving sign } & $14(48.2)$ & Irregular pleural line & $14(100)$ & Crazy paving sign & $17(56.7)$ & Irregular pleural line & $17(100)$ & 0.444 & 0.574 \\
\hline & & Compound B lines & $12(85.7)$ & & & Compound B lines & $14(82.4)$ & & 0.423 \\
\hline & & Diffuse B lines & $7(50.0)$ & & & Diffuse B lines & $8(47.1)$ & & 0.854 \\
\hline \multirow[t]{2}{*}{ Subpleural lines } & $8(27.5)$ & Irregular pleural line & $8(100)$ & Subpleural lines & $10(33.3)$ & Irregular pleural line & $10(100)$ & 0.205 & 0.886 \\
\hline & & Compound B lines & $8(100)$ & & & Compound B lines & $8(80.0)$ & & 0.543 \\
\hline Pleural effusion & $1(3.4)$ & Pleural effusion & $1(100)$ & Pleural effusion & $1(3.4)$ & Pleural effusion & $1(100)$ & 0.823 & - \\
\hline Consolidations & $5(17.2)$ & Consolidations & $4(80.0)$ & Consolidations & $5(0.8)$ & Consolidations & $5(100)$ & - & - \\
\hline
\end{tabular}

CT, computed tomography; LUS, lung ultrasonography; Compound B lines, less than $50 \%$ of the intercostal space; diffuse B lines, white lung.

${ }^{\text {a) }}$ Comparison of CT findings of the 12-zone and 14-zone study groups. ${ }^{\mathrm{b})}$ Comparison of LUS findings of the 12-zone and 14-zone study groups. 


\section{Discussion}

A moderate and high degree of agreement was found between LUS and thoracic CT scores using 12 and 14 zones, respectively. The ground-glass appearance detected on $\mathrm{CT}$ can be screened for using both imaging protocols with findings such as pleural lines and B-line abnormalities in LUS.

Three major radiological methods can be used in the diagnosis and follow-up of COVID-19 pneumonia. Among these methods, the routine use of chest radiography has not found a major place in clinical practice during the pandemic, since it has weaknesses in the early-stage diagnosis of COVID-19 [14]. CT, the second method, is considered to be the most sensitive technique for diagnosing the disease at an early stage, evaluating the size and morphology of the detected lesions, and identifying small lesions that cannot be

Table 3. CT and LUS scores according to the evaluation areas

\begin{tabular}{lccc}
\hline & CT score & LUS score & P-value $^{\text {a) }}$ \\
\hline Total score & & & \\
12 Areas $(n=29)$ & $12.9 \pm 6.3$ & $18.4 \pm 7.4$ & $P<0.001, r=0.827$ \\
14 Areas $(n=30)$ & $11.9 \pm 6.3$ & $21.0 \pm 7.7$ & $P<0.001, r=0.840$ \\
P-value ${ }^{b)}$ & 0.215 & & \\
Right lung & & & \\
12 Areas $(n=29)$ & $7.1 \pm 3.2$ & $10.3 \pm 3.9$ & $P<0.001, r=0.707$ \\
14 Areas $(n=30)$ & $7.0 \pm 3.7$ & $11.8 \pm 3.5$ & $P<0.001, r=0.849$ \\
P-value ${ }^{b)}$ & 0.128 & & \\
Left lung $_{\text {12 Areas }(n=29)}$ & $5.8 \pm 2.9$ & $8.0 \pm 3.7$ & $P<0.001, r=0.808$ \\
14 Areas $(n=30)$ & $3.9 \pm 2.5$ & $9.2 \pm 4.6$ & $P<0.001, r=0.954$ \\
P-value ${ }^{b)}$ & 0.013 & & \\
\hline
\end{tabular}

Values are presented as mean \pm standard deviation.

CT, computed tomography; LUS, lung ultrasonography.

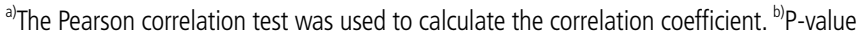
from the independent-samples t-test. detected on chest radiography [15]. LUS, as the third method, has been reported to have a high sensitivity for diagnosing pulmonary interstitial involvement associated with COVID-19 [16,17]. When compared to $\mathrm{CT}$, the main reason for its high sensitivity is thought to be the ability of LUS to detect the subpleural lesions in COVID-19 pneumonia, especially involvement in the lower and middle lobe of the lung $[16,17]$. In the literature, studies have used the BLUE

Table 4. CT and LUS scores by lobes in the evaluation areas

\begin{tabular}{|c|c|c|c|}
\hline & CT score & LUS score & $P$-value ${ }^{a)}$ \\
\hline \multicolumn{4}{|l|}{ Right upper lobe } \\
\hline 12 Areas $(n=29)$ & $1.0 \pm 1.2$ & $3.4 \pm 7.5$ & $P<0.001, r=0.787$ \\
\hline 14 Areas $(n=30)$ & $1.2 \pm 0.9$ & $2.8 \pm 2.0$ & $P<0.001, r=0.933$ \\
\hline$P$-value & 0.878 & & \\
\hline \multicolumn{4}{|l|}{ Right middle lobe } \\
\hline 12 Areas $(n=29)$ & $3.2 \pm 1.1$ & $2.5 \pm 0.9$ & $P<0.001, r=0.630$ \\
\hline 14 Areas $(n=30)$ & $3.1 \pm 1.4$ & $2.4 \pm 1.4$ & $P<0.001, r=0.757$ \\
\hline P-value & 0.568 & & \\
\hline \multicolumn{4}{|l|}{ Right lower lobe } \\
\hline 12 Areas $(n=29)$ & $3.1 \pm 1.4$ & $3.7 \pm 7.0$ & $P=0.024, r=0.518$ \\
\hline 14 Areas $(n=30)$ & $3.6 \pm 1.4$ & $5.9 \pm 0.4$ & $P=0.015, r=0.538$ \\
\hline P-value & 0.648 & & \\
\hline \multicolumn{4}{|l|}{ Left upper lobe } \\
\hline 12 Areas $(n=29)$ & $3.4 \pm 1.5$ & $2.6 \pm 2.7$ & $P<0.001, r=0.902$ \\
\hline 14 Areas $(n=30)$ & $3.4 \pm 1.3$ & $3.2 \pm 2.6$ & $P<0.001, r=0.850$ \\
\hline P-value & 0.205 & & \\
\hline \multicolumn{4}{|l|}{ Left lower lobe } \\
\hline 12 Areas $(n=29)$ & $2.4 \pm 1.6$ & $5.0 \pm 3.91$ & $P=0.020, r=0.521$ \\
\hline 14 Areas $(n=30)$ & $1.4 \pm 1.2$ & $6.9 \pm 2.4$ & $P<0.001, r=0.902$ \\
\hline P-value & 0.019 & & \\
\hline
\end{tabular}

Values are presented as mean \pm standard deviation.

$\mathrm{CT}$, computed tomography; LUS, lung ultrasonography.

${ }^{a}$ The Pearson correlation test was used to calculate the correlation coefficient. ${ }^{\text {b) }}$-value from the independent-samples t-test.

Table 5. Correlations of the LUS scores in the 12-zone and 14-zone groups with physical examination findings and laboratory results

\begin{tabular}{|c|c|c|c|c|}
\hline & $\begin{array}{c}\text { LUS score } \\
12 \text { zones }(n=29)\end{array}$ & P-value ${ }^{a)}$ & $\begin{array}{c}\text { LUS score } \\
14 \text { zones }(n=30)\end{array}$ & P-value ${ }^{a)}$ \\
\hline LUS score & $21.0 \pm 7.7$ & & $18.4 \pm 7.4$ & \\
\hline APACHE II score & $23.8 \pm 5.3$ & $P<0.001 \quad r=0.656$ & $22.4 \pm 5.6$ & $P<0.001 \quad r=0.685$ \\
\hline Ferritin (ng/mL) & $1,126 \pm 326$ & $P<0.001 \quad r=0.703$ & $1,093 \pm 298$ & $P<0.001 \quad r=0.727$ \\
\hline C-reactive protein (mg/dL) & 90.3 & $P<0.001 \quad r=0.691$ & 86.4 & $P<0.001 \quad r=0.679$ \\
\hline $\mathrm{SpO}_{2}(\%)$ mean & 91.0 & $P<0.001 \quad r=0.806$ & 91.2 & $P<0.001 \quad r=0.842$ \\
\hline Lymphocytes $\left(\times 10^{9} / \mathrm{L}\right)$ & 0.58 & $P<0.001 \quad r=0.925$ & 0.61 & $P<0.001 \quad r=0.950$ \\
\hline
\end{tabular}

Values are presented as mean \pm standard deviation unless otherwise indicated.

LUS, lung ultrasonography; APACHE, Acute Physiology and Chronic Health Evaluation; $\mathrm{SpO}_{2}$, saturated oxygen.

${ }^{a}$ The Pearson correlation test was used to calculate the correlation coefficient. 


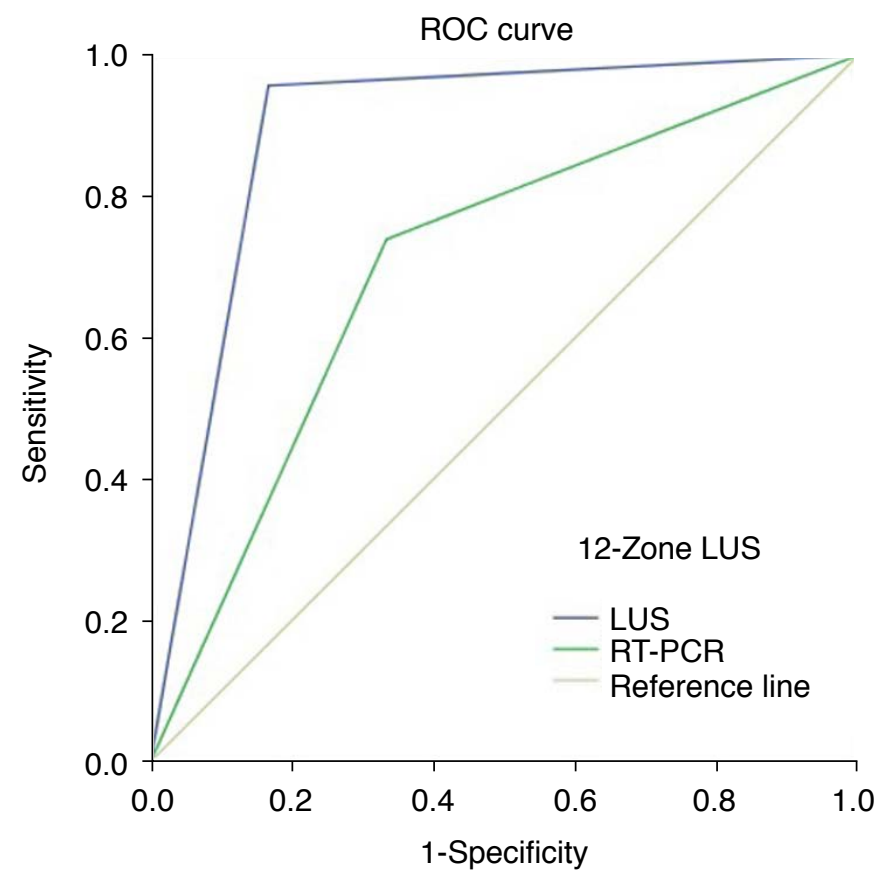

A

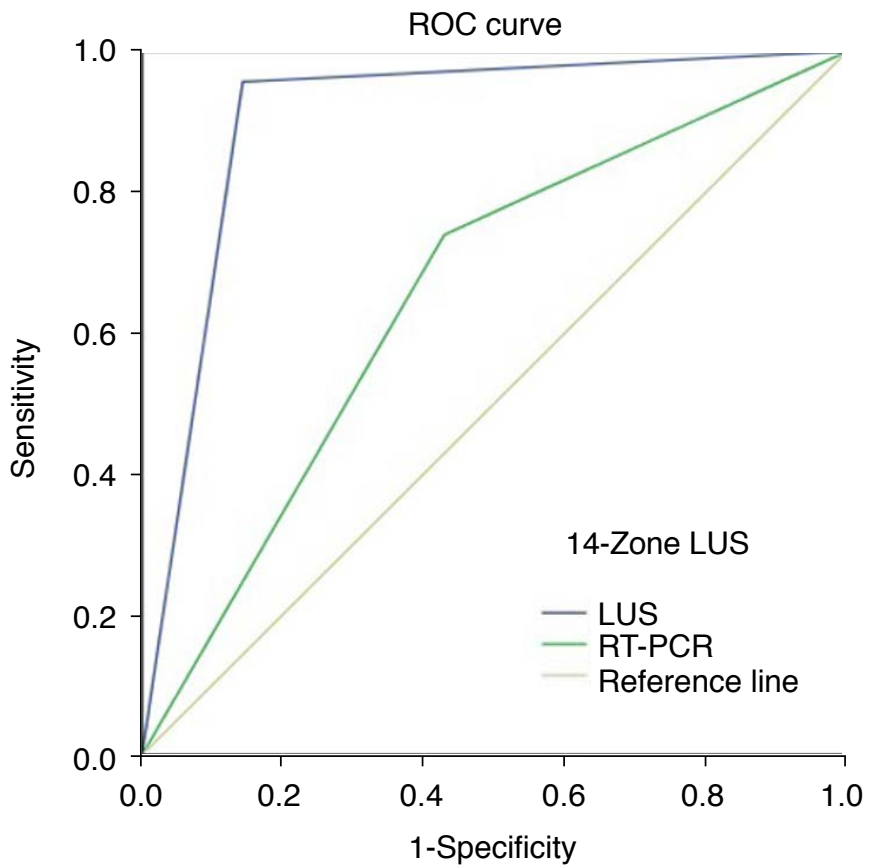

B

Fig. 5. Receiver operating characteristic curve of reverse-transcription polymerase chain reaction (RT-PCR) test and lung ultrasonography (LUS) for detecting computed tomography abnormalities.

A. In 12-zone group, area under the curve (AUC) of LUS (0.895) is higher than that of RT-PCR (0.703). B. In 14-zone group, AUC of LUS (0.908) is also higher than that of RT-PCR (0.655).

protocol and modified versions of LUS in the diagnosis and followup of pulmonary involvement of COVID-19 [8]. The differences in the examination zones used in these protocols are noteworthy. Similar results were obtained in studies of LUS examinations performed with different protocols in previously defined zones of the lung. Methods involving $8,10,12$, and 14 zones have been used to examine the anterior, lateral, and posterior lung areas in the thoracic wall $[8,9,16,17]$.

Dacrema et al. [18] used a rapid and simplified scanning method referred to as LUSCOP, which includes six regions (apex, middle, and basal), to evaluate 130 patients. They reported an achievement rate of $99.2 \%$ in identifying COVID-19 pneumonia [18]. Other research using the 12-zone examination method compared the diagnostic success of LUS to CT [19]. The mean LUS score was determined to be 10.6 in a study that included 51 patients. In the same study, the CT score was calculated as 7.48 , and its correlation with the LUS score was found to be 0.803 . The researchers reported that the LUS findings observed in their study included pleural line and B-line abnormalities, similar to the results of other studies [19]. In a study using the same number of examination zones in intensive care patients, the correlation between LUS scores and CT scores was 0.87 , and it was found that there was a good degree of agreement [20]. In addition, the researchers reported that LUS yielded similar results regardless of whether six or 12 zones were evaluated. Although these results need to be confirmed, the researchers stated that the results were similar to data in the literature and the results of studies using the BLUE protocol [20]. Due to the presence of studies in the literature using different methods of examination, Soldati et al. created a LUS protocol with the aim of developing international standardization and presented their opinions about a 14-zone imaging method as part of their protocol. It was emphasized that the imaging method should definitely include the posterior regions, since the posterior lung is more extensively involved in COVID-19 pneumonia [9]. Mento et al. investigated the results of different protocols when administered to the same patients. They analyzed the results of LUS protocols involving $4,8,12$, and 14 zones in data obtained from 88 patients, who had been diagnosed with COVID-19, and reported that the 12-zone LUS protocol achieved a good balance between the duration of the procedure and accuracy. They found that the inclusion of posterior areas in the protocols increased the level of agreement between the modified systems. As mentioned in the literature, they emphasized the importance of examining the posterior areas in order to detect important findings in patients with COVID-19 [21]. A study analyzing a protocol using 14 landmarks during the admission and follow-up of intensive care patients reported that this protocol could be used in intensive care 
units to predict the clinical course of COVID-19 pneumonia and decide upon a treatment strategy [22].

In the present study, a score was calculated for each lobe by matching the lung lobes with the imaging zones. The authors suggest that this strategy enabled a more detailed examination of the scores and lesions of each lobe. The 14-zone examination has a greater scope for the evaluation of the lesions in the lower lobe of the lung. The higher incidence of COVID-19 pneumonia in the lower lung lobes is the reason why this method is advantageous [9]. In addition, despite the lower $\mathrm{CT}$ scores detected in the left lower lobe in patients who underwent 14-zone examinations, higher correlation values were found with the LUS scores in the same zones. This finding supports the view that has been presented in the literature that reducing the number of imaging zones in patients affected by COVID-19 would increase the probability of missing important findings $[23,24]$. In diseases with a high risk of transmission such as COVID-19, the balance between the duration of the procedure and the level of data collection during LUS can make it difficult for clinicians to decide which protocol to use. We believe that further data will clarify the principles of the LUS procedure over time.

The fact that LUS examinations were performed only during hospitalization led the researchers to select only patients diagnosed with COVID-19 who were receiving treatment in the intensive care unit. The limitations of this study include the general weaknesses of ultrasonography such as its technical characteristics, the variability depending on the specialist responsible for performing LUS, and the use of only a convex probe during the examination. In addition, since LUS was performed one time in each patient by a single user, the inability to measure intra- and inter-observer variability is a limitation.

The results of our study indicated that the two different LUS examination methods performed in different patients had similar findings in terms of the diagnosis and their correlations with CT results. Extensive data in the literature have been reported regarding the role of LUS in the diagnosis of pneumonia associated with COVID-19. The authors suggest that the method to be selected and the procedure will become clearer with further studies and the inclusion of learning-based systems [11].

ORCID: Korgün Ökmen: https://orcid.org/0000-0001-8546-4661; Durdu Kahraman Y.ldı: https://orcid.org/0000-0003-1035-0306; Emel Soyaslan: https://orcid.org/00000003-4343-6616; Illkay Ceylan: https://orcid.org/0000-0003-3306-3107; Halil Erkan Sayan: https://orcid.org/0000-0003-3943-5549; Cihan Sedat Aytünür: https://orcid.org/0000-00019342-6710

\section{Author Contributions}

Conceptualization: Ökmen K, Yıldız DK, Ceylan İ, Aytünür CS. Data acquisition: Ökmen K, Sayan HE. Data analysis or interpretation: Ökmen K, Soyaslan E. Drafting of the manuscript: Ökmen K, Yıldız DK, Soyaslan E, Sayan HE, Aytünür CS. Critical revision of the manuscript: Ceylan i. Approval of the final version of the manuscript: all authors.

\section{Conflict of Interest}

No potential conflict of interest relevant to this article was reported.

\section{Supplementary Material}

Supplementary Data 1. Inspection zones (https://doi.org/10.14366/ usg.21095).

\section{References}

1. Cucinotta D, Vanelli M. WHO declares COVID-19 a pandemic. Acta Biomed 2020;91:157-160.

2. Wujtewicz M, Dylczyk-Sommer A, Aszkielowicz A, Zdanowski S, Piwowarczyk S, Owczuk R. COVID-19: what should anaethesiologists and intensivists know about it? Anaesthesiol Intensive Ther 2020;52:34-41.

3. Chung M, Bernheim A, Mei $X$, Zhang $N$, Huang $M$, Zeng $X$, et al. CT imaging features of 2019 novel coronavirus (2019-nCoV). Radiology 2020;295:202-207.

4. Xu B, Xing Y, Peng J, Zheng Z, Tang W, Sun Y, et al. Chest CT for detecting COVID-19: a systematic review and meta-analysis of diagnostic accuracy. Eur Radiol 2020;30:5720-5727.

5. Ye Z, Zhang Y, Wang Y, Huang Z, Song B. Chest CT manifestations of new coronavirus disease 2019 (COVID-19): a pictorial review. Eur Radiol 2020;30:4381-4389.

6. Zhao L, Yu K, Zhao Q, Tian R, Xie H, Xie L, et al. Lung ultrasound score in evaluating the severity of coronavirus disease 2019 (COVID-19) pneumonia. Ultrasound Med Biol 2020;46:2938-2944.

7. Smith MJ, Hayward SA, Innes SM, Miller AS. Point-of-care lung ultrasound in patients with COVID-19: a narrative review. Anaesthesia 2020;75:1096-1104.

8. Sultan LR, Sehgal CM. A Review of early experience in lung ultrasound in the diagnosis and management of COVID-19. Ultrasound Med Biol 2020;46:2530-2545.

9. Soldati $G$, Smargiassi $A$, Inchingolo R, Buonsenso D, Perrone $T$, Briganti DF, et al. Proposal for international standardization of the use of lung ultrasound for patients with COVID-19: a simple, quantitative, reproducible method. J Ultrasound Med 2020;39:1413-1419.

10. Okmen K, Yildiz DK, Soyaslan E. Comparison of lung ultrasonography findings with chest computed tomography results in coronavirus (COVID-19) pneumonia. J Med Ultrason (2001) 2021;48:245-252. 
11. Chang YC, Yu CJ, Chang SC, Galvin JR, Liu HM, Hsiao CH, et al. Pulmonary sequelae in convalescent patients after severe acute respiratory syndrome: evaluation with thin-section CT. Radiology 2005;236:1067-1075.

12. Soummer A, Perbet $S$, Brisson $H$, Arbelot $C$, Constantin JM, Lu Q, et al. Ultrasound assessment of lung aeration loss during a successful weaning trial predicts postextubation distress. Crit Care Med 2012;40:2064-2072.

13. Rouby JJ, Arbelot C, Gao Y, Zhang M, Lv J, An Y, et al. Training for lung ultrasound score measurement in critically ill patients. Am J Respir Crit Care Med 2018;198:398-401.

14. Yang W, Sirajuddin A, Zhang X, Liu G, Teng Z, Zhao S, et al. The role of imaging in 2019 novel coronavirus pneumonia (COVID-19). Eur Radiol 2020;30:4874-4882.

15. Jin YH, Cai L, Cheng ZS, Cheng H, Deng T, Fan YP, et al. A rapid advice guideline for the diagnosis and treatment of 2019 novel coronavirus (2019-nCoV) infected pneumonia (standard version). Mil Med Res 2020;7:4.

16. Campagnano S, Angelini F, Fonsi GB, Novelli S, Drudi FM. Diagnostic imaging in COVID-19 pneumonia: a literature review. J Ultrasound 2021;24:383-395.

17. Dudea SM. Ultrasonography and SARS-CoV 2 infection: a review of what we know and do not yet know. Med Ultrason 2020;22:129132.

18. Dacrema A, Silva M, Rovero L, Vertemati V, Losi G, Piepoli MF, et al. A simple lung ultrasound protocol for the screening of COVID-19 pneumonia in the emergency department. Intern Emerg Med 2021;16:1297-1305.

19. Tung-Chen Y, Marti de Gracia M, Diez-Tascon A, Alonso-Gonzalez R, Agudo-Fernandez S, Parra-Gordo ML, et al. Correlation between chest computed tomography and lung ultrasonography in patients with coronavirus disease 2019 (COVID-19). Ultrasound Med Biol 2020;46:2918-2926.

20. Heldeweg ML, Lopez Matta JE, Haaksma ME, Smit JM, Elzo Kraemer CV, de Grooth $\mathrm{HJ}$, et al. Lung ultrasound and computed tomography to monitor COVID-19 pneumonia in critically ill patients: a two-center prospective cohort study. Intensive Care Med Exp 2021;9:1.

21. Mento F, Perrone T, Macioce VN, Tursi F, Buonsenso D, Torri E, et al. On the impact of different lung ultrasound imaging protocols in the evaluation of patients affected by coronavirus disease 2019: how many acquisitions are needed? J Ultrasound Med 2020;40:22352238.

22. Perrone T, Soldati G, Padovini L, Fiengo A, Lettieri G, Sabatini U, et al. A new lung ultrasound protocol able to predict worsening in patients affected by severe acute respiratory syndrome coronavirus 2 pneumonia. J Ultrasound Med 2021;40:1627-1635.

23. Demi L. Lung ultrasound: the future ahead and the lessons learned from COVID-19. J Acoust Soc Am 2020;148:2146.

24. Smargiassi A, Soldati G, Torri E, Mento F, Milardi D, Del Giacomo P, et al. Lung ultrasound for COVID-19 patchy pneumonia: extended or limited evaluations? J Ultrasound Med 2021;40:521-528. 